\title{
The clinical value of new diagnostic tools for tuberculosis Andrew Ramsay ${ }^{*}$ and Anthony D Harries ${ }^{2,3}$
}

\author{
Address: ${ }^{1}$ UNICEF/United Nations Development Programme/World Bank/World Health Organization Special Programme for Research and \\ Training in Tropical Diseases, World Health Organization, 20 Avenue Appia, 1211 Geneva 27, Switzerland; ${ }^{2}$ International Union Against \\ Tuberculosis and Lung Disease, 68 Boulevard Saint-Michel, 75006 Paris, France; ${ }^{3}$ London School of Hygiene and Tropical Medicine, \\ Keppel Street, London WC1E 7HT, UK \\ * Corresponding author: Andrew Ramsay (ramsaya@who.int) \\ FI000 Medicine Reports 2009, I:36 (doi:I0.3410/MI-36)
}

The electronic version of this article is the complete one and can be found at: http://FI000.com/Reports/Medicine/content/I/36

\begin{abstract}
Barriers to global tuberculosis (TB) control include multidrug resistance, HIV infection, and weak health systems. Case detection is critical to TB control and is affected by all three of these. Currently, most low- and middle-income countries (LMICs) rely on direct sputum smear microscopy for diagnosis. Modern culture methods and molecular tests, previously considered too complex or too expensive for implementation in LMICs, are now being introduced there in parallel with a global effort to strengthen laboratories. It remains to be seen whether services based on these tools can be made widely accessible to patients. New point-of-care tests for TB are urgently needed but cannot be expected in the near future. In the meantime, diagnostic tools based on optimized smear microscopy, although less sensitive than reference laboratory tests, may be more accessible and have more impact on case finding. It is a matter of urgency that these improved microscopy services be integrated with services based on rapid methods that can identify multidrug-resistant cases.
\end{abstract}

\section{Introduction and context}

In 2006, there were an estimated 9.2 million new cases of tuberculosis (TB), the majority occurring in low- and middle-income countries (LMICs) [1]. Diagnosing TB on the basis of clinical and radiological findings alone is known to be inaccurate, particularly in HIV-associated $\mathrm{TB}[2,3]$. The definitive diagnosis is bacteriological. Most LMICs rely almost entirely on direct sputum smear microscopy (DSSM) for routine TB diagnostic services. This involves the examination of a series of sputum specimens from each patient and requires repeated patient visits to health facilities to submit specimens and to collect results. International guidelines exist for the DSSM-based diagnosis and management of TB suspects, and patients and most countries have adopted these in their national programmes [4,5]. International efforts to control TB, largely based on DSSM, ensure that millions of patients receive treatment and hundreds of thousands of lives are saved each year, but so far these efforts have failed to substantially reduce the annual global incidence [1]. Three major barriers to global TB control have been identified: the weak health systems that exist in many countries, the growing resistance of Mycobacterium tuberculosis (MTB) to the currently used anti-TB drugs, and HIV infection [1].

It is also recognized that inadequate case finding is a major obstacle to global TB control [1]. This inadequacy may be considered both quantitative and qualitative. Case finding may be inadequate, quantitatively, in failing to identify the majority of those in the community with TB. In many countries in recent decades, HIV has compromised quantitative case finding by altering the clinical presentation of $\mathrm{TB}$ and mitigating the immunological reaction to infection, which in turn results in a lower sputum bacillary load [6]. Case finding may also be considered qualitatively inadequate in failing to distinguish between TB cases with and without critical patterns of drug resistance that impact upon treatment success and continued transmission. Both forms of 
inadequate case finding are exacerbated by the weak or frankly broken health systems that exist in many countries. They are also exacerbated by widespread poverty in LMICs. Many poor people who need to be investigated for TB are unable to afford repeated visits to health facilities for smear diagnosis, and frequently default during the diagnosis process [7]. Services, based on new tools, that can be delivered within resource-poor health systems, that are sensitive to the poverty of many service users, and that result in the increased identification of HIV-associated and drug-resistant TB cases, could make a major contribution to global TB control.

\section{Recent advances Current tools for diagnosing TB in resource-poor settings}

Currently, all bacteriological diagnostic tests for TB rely upon microscopically visualizing the characteristic acidfast bacilli in specially stained sputum smears, growing and identifying MTB in cultures of specimens, or detecting MTB-specific nucleic acids in specimens. Diagnosis through MTB culture or nucleic acid detection is more sensitive than DSSM, and particularly so in HIV-associated $\mathrm{TB}$, in which DSSM is notoriously insensitive [8]. These techniques also have the benefit of making isolates or nucleic acids available for drug susceptibility testing. A disadvantage of these tests is that they take considerably longer than smear microscopy for a result to be available for the management of the patient. This may be because the test itself takes several weeks to complete (culture) and/or because it requires a sophisticated bio-safe laboratory and, unavoidably, a centralized service of some kind. Centralized services and the logistics involved in specimen transport and delivering laboratory reports within a clinically useful time frame are particularly difficult to organize within weak health systems $[9,10]$. Where these tests have been introduced at the National TB Reference Laboratory (NRL) level, they have been associated with limited impact on TB case management [10].

\section{Integrating new TB diagnostic tools in resource-poor settings}

Until recently, modern culture methods and nucleic acid detection tests have been considered either too complex or too expensive for implementation in LMICs outside of NRLs. In the past two years, the World Health Organization has endorsed the use of both liquid culture systems (plus new rapid methods for identifying isolates) and molecular line-probe assays for TB control in LMICs $[11,12]$. There are now considerable global efforts under way to assist National TB Programs (NTPs) in LMICs to build laboratory capacity to introduce these new tools and develop services based on them [13]. It is recognized that that there is no strong evidence that the introduction of these tools will actually improve TB control at the routine programmatic level. Field studies and costeffectiveness data are needed to better understand the real-world implications of the changes [14]. There are considerable challenges involved in delivering services based on these technologies in LMICs. These challenges are well recognized, but with little prospect that technology platforms will become available in the near future and thus obviate the need for greatly increased laboratory capability/capacity, there is an imperative to act now.

The Retooling Task Force and the New Diagnostics Working Group of the Stop TB Partnership recently described the pipeline of new diagnostic tools for TB [15]. Of eight new tools considered to be in late-stage development and perhaps available within the next few years, one is a nucleic acid detection test (which may be simpler than current line-probe assays for drug resistance detection) and four are culture-based diagnostics. Of the remaining three tools in late-stage development, two are based on improved smear microscopy. The remaining tool is the interferon-gamma release assay, which (though available on the market) has not yet been endorsed by the World Health Organization for use in TB control programmes, as there is considerable uncertainty about its likely contribution to case finding in LMICs [16]. The two improved microscopy tools, being appropriate for the lower levels of poor health services, may have considerably more impact on quantitative case finding in LMICs than either the culture methods or the molecular assays. One of the improved microscopy tools is fluorescent microscopy (FM) systems based on inexpensive battery-powered light-emitting diodes (LEDs) for DSSM [17]. In a recent systematic review, FM was found to have comparable specificity to ZiehlNeelsen DSSM but with an approximately 10\% increase in sensitivity while taking around $25 \%$ of the time to examine smears [18]. The benefits for case finding and alleviating heavy workloads in laboratories have not been realizable to date since conventional fluorescence microscopes were complex and very expensive. The LEDFM systems are currently under evaluation in a number of LMICs. The other improved microscopy tool, frontloaded microscopy, is an approach rather than a technological change [19]. A systematic review of the yield of serial sputum specimens has reported that the first two specimens (collected as spot and morning) identify 95-98\% of all smear-positive cases [20]. Because a considerable proportion of patients default from the current DSSM diagnostic process (that requires multiple patient visits), front-loaded smear microscopy involves collecting and examining two sputum specimens on the first day a patient presents and referring those patients in whom the sputum is smear-positive immediately for 
treatment. Multi-country trials of front-loaded microscopy are ongoing. These optimized smear microscopy tools, though less sensitive than reference laboratory tests, may be more accessible and have a greater public health impact [21]. However, they will not identify drug resistance.

\section{Implications for clinical practice Using new tools to improve diagnostic services for $T B$ in resource-poor settings}

Diagnostic services based on new tools, whether new (or modified) technologies or new approaches to delivery, have the potential to revolutionize TB case finding. The deficiencies in both quantitative and qualitative case findings need to be addressed. Diagnostic services need to identify more $\mathrm{TB}$ cases and to identify drug-resistant cases. Such services are unlikely, in the foreseeable future, to be based upon the introduction of a single new diagnostic tool. Rather, they will involve multiple tools being implemented in an integrated way within a tiered health system [15]. The new diagnostic tools, as well as being integrated with the health systems, will need to be carefully integrated with algorithms for the clinical management of cases. Simple new tools for the diagnosis of pulmonary $\mathrm{TB}$ at the lowest levels of health services (point-of-care) and for the diagnosis of extrapulmonary and childhood TB are also urgently needed. They are not yet on the horizon.

\section{Abbreviations}

DSSM, direct sputum smear microscopy; FM, fluorescent microscopy; LED, light-emitting diode; LMIC, low- and middle-income country; MTB, Mycobacterium tuberculosis; NRL, National Tuberculosis Reference Laboratory; NTP, National TB Program; TB, tuberculosis.

\section{Competing interests}

The authors declare that they have no competing interests.

\section{References}

I. World Health Organization: Global Tuberculosis Control: Surveillance, Planning, Financing. WHO Report 2008. Geneva, Switerland: WHO; 2008 (WHO/HTM/TB/2008.393). [http://www.who.int/tb/publications/global_report/en]

2. Nyirenda TE, Harries AD, Banerjee A, Salaniponi FM: Accuracy of chest radiograph diagnosis for smear-negative pulmonary tuberculosis suspects by hospital clinical staff in Malawi. Trop Doct 1999, 29:219-20.

3. Colebunders $R$, Bastian I: A review of the diagnosis and treatment of smear-negative pulmonary tuberculosis. Int J Tuberc Lung Dis 2000, 4:97-107.

4. World Health Organization: Treatment of Tuberculosis: Guidelines for National Programmes. 3rd edition. Geneva, Switzerland: WHO; 2003 (WHO/CDS/TB/2003.3/3).

5. Enarson DA, Rieder HL, Arnadottir T, Trébucq A: Management of Tuberculosis: A Guide for Low Income Countries. 5th edition. Paris: International Union Against Tuberculosis and Lung Disease; 2000.
6. Mugusi F, Villamor E, Urassa W, Saathoff E, Bosch RJ, Fawzi WW: HIV co-infection, CD4 cell counts and clinical correlates of bacillary density in pulmonary tuberculosis. Int J Tuberc Lung Dis 2006, 10:663-9.

7. Kemp JR, Mann GH, Nhlema-Simwaka B, Salaniponi FML, Squire SB: Can Malawi's poor afford free TB services? Patient and household costs associated with TB diagnosis in Lilongwe. Bull World Health Organ 2007, 85:580-5.

FI000 Factor 3.0 Recommended Evaluated by Anthony Harries 16 Oct 2007

8. Perkins MD, Cunningham J: Facing the crisis: improving the diagnosis of tuberculosis in the HIV era. J Inf Dis 2007, 196:SI 15-27.

9. Yagui M, Perales MT, Asencios L, Vergara L, Suarez C, Yale G, Salazar C, Saavedra M, Shin S, Ferrousier O, Ciegelski P: Timely diagnosis of MDR-TB under program conditions: is rapid drug susceptibility testing sufficient? Int J Tuberc Lung Dis 2006, 10:838-43.

10. Harries AD, Michongwe J, Nyirenda TE, Kemp JR, Squire SB, Ramsay AR, Godfrey-Faussett P, Salaniponi FML: Using a bus service for transporting sputum specimens to the Central Reference Laboratory: effect on the routine TB culture service in Malawi. Int J Tuberc Lung Dis 2004, 8:204-I0.

II. World Health Organization: The use of liquid medium for culture and DST. [http://www.who.int/tb/dots/laboratory/policy/en/ index3.html]

12. World Health Organization: WHO Policy Statement: molecular line probe assays for rapid screening of patients at risk of multidrug-resistant tuberculosis (MDR-TB). 27 June 2008. [http://www.who.int/tb/features_archive/policy_statement.pdf]

13. World Health Organization: Strengthening tuberculosis laboratories: case detection through quality-assured bacteriology. [http://www.who.int/tb/dots/laboratory/en/index.html]

14. Pai M, Ramsay A, O'Brien R: Evidence-based tuberculosis diagnosis. PLoS Medicine 2008, 5:el 56.

15. World Health Organization: New laboratory diagnostic tools for tuberculosis control. Geneva, Switzerland: WHO Press, 2008. [http://www.stoptb.org/retooling/assets/documents/Diagnostic Brochure_Final_Dec_08.pdf]

16. Barth RE, Mudrikova T, Hoepelman Al: Interferon-gamma release assays (IGRAs) in high-endemic settings: could they play a role in optimizing global TB diagnostics. Evaluating the possibilities of using IGRAs to diagnose active TB in a rural African setting. Int J Infect Dis 2008, 12:el-6.

17. Marais BJ, Brittle W, Painczyk K, Hesseling AC, Beyers N, Wasserman $E$, van Soolingen $D$, Warren RM: Use of light-emitting diode fluorescence microscopy to detect acid-fast bacillin in sputum. Clin Infect Dis 2008, 47:203-7.

18. Steingart KR, Henry M, Ng V, Hopewell PC, Ramsay A, Cunningham J, Urbanczik R, Perkins M, Aziz MA, Pai M: Fluorescence versus conventional sputum smear microscopy for tuberculosis: a systematic review. Lancet Infect Dis 2006, 6:570-81.

19. Ramsay A, Yassin MA, Cambanis A, Hirao S, Almotawa A, Gammo M, Lawson L, Arbide I, Al-Aghbari N, Al-Sonboli N, Serchand JB, Gauchan P, Cuevas LE: Front-loading sputum microscopy services: an opportunity to optimise smear-based casedetection of tuberculosis in high prevalence countries. Journal of Tropical Medicine 2009, in press.

20. Mase SR, Ramsay A, Henry M, Ng V, Hopewell PC, Cunningham J, Urbanczik R, Perkins M, Aziz MA, Pai M: Yield of serial sputum specimen examinations in the diagnosis of pulmonary tuberculosis: a systematic review. Int J Tuberc Lung Dis 2007, I I:485-95.

21. Keeler E, Perkins MD, Small P, Hanson C, Reed S, Cunningham J, Aledort JE, Hillborne L, Rafael ME, Girosi F, Dye C: Reducing the global burden of tuberculosis: the contribution of improved diagnostics. Nature 2006, 444(SuppI I):49-57. 